\title{
Comment on: Sentinel Node Biopsy Using Magnetic Tracer Versus Standard Technique: The SentiMAG Multicentre Trial
}

\author{
Emmanuel Barranger, MD, PhD and Tarik Ihrai, MD \\ Breast and Gynecological Surgical Oncology Unit, Centre Antoine Lacassagne, University of Nice Sophia-Antipolis, Nice \\ Cedex 2, France
}

\section{TO THE EDITORS:}

The standard practice in breast cancer surgery is to mandate a sentinel node biopsy (SNB) for all patients without clinically negative axillary lymph node. To date, the "gold standard" for detecting the sentinel lymph node (SLN) is a combination of blue dye and radioisotope. ${ }^{1}$ However, in recent years, the use of these tracers has been controversial because of allergic complications involving blue dye and organizational difficulties regarding the radioisotopic method. This has led some teams to develop new tracers for SLN localization. Thus, a magnetic tracer was recently developed to replace potentially one of two commonly used tracers.

We read with interest the recently published study by Douek et al., which evaluated in a multicenter trial the performance of a new magnetic technique for SNB. ${ }^{2}$ In a large series of patients $(N=170)$, the authors evaluated a magnetic tracer against the standard detection technique (radioisotope with or without blue dye). The main results of this prospective study showed an excellent identification rate using the magnetic method $(94.4 \%)$ and a low discordance rate $(6.9 \%)$ between the standard technique and the magnetic method. The authors concluded that this technique is feasible for SNB and recommended a randomized, controlled trial to validate the magnetic technique.

The results of this study are interesting. However, the authors do not sufficiently discuss technical issues and surgical difficulties related to the use of this new tracer. We

(C) Society of Surgical Oncology 2017

First Received: 13 October 2017;

Published Online: 22 November 2017

E. Barranger, MD, $\mathrm{PhD}$

e-mail: emmanuel.barranger@nice.unicancer.fr agree that the standard technique has drawbacks, such as radiation exposure of patients and healthcare personnel, strict legislative control, limitations on radiotracer availability, dependency on nuclear medicine units, and allergic reactions to blue dye. In addition, it is our view that the use of this tracer presents a number of disadvantages. We consider that this tracer is not easy to use for surgeons and requires a minimum learning curve. The main technical disadvantage of this procedure is the large diameter of the handheld magnetometer, which makes it necessary to enlarge the incision to insert the probe and to identify the magnetic SLN. Second, identification of the magnetic SLN requires the probe to be in contact with the lymph node and for surgical instruments to contain no iron. Finally, the handheld magnometer requires regular calibration during usage, thus lengthening the duration of surgery.

In conclusion, even if magnetic detection offers certain advantages, we must not forget that this detection method has many drawbacks that require technical improvement before it can be applied in clinical practice.

DISCLOSURE The authors reports that they have no conflict of interest.

\section{REFERENCES}

1. Kim T, Giuliano AE, Lyman GH. Lymphatic mapping and sentinel lymph node biopsy in early-stage breast carcinoma. Cancer. 2006;106:4-16.

2. Douek M, Klaase J, Monypenny I, Kothari A, Zechmeister K, Brown D, et al; On behalf of the SentiMAG Trialists Group. Sentinel node biopsy using a magnetic tracer versus standard technique: the SentiMAG Multicentre Trial. Ann Surg Oncol. 2013;21(4):1237-45. 